\title{
A Fast Single Cut Spherical Near-Field-to-Far-Field Transformation Using Cylindrical Modes
}

\author{
T. Salmerón-Ruiz , M. Sierra-Castañer , F. Saccardi , S. Burgos , F. J. Cano-Fácila, Lars J. Foged
}

\begin{abstract}
A fast spherical near-field to far-field transformation using single cuts is introduced in this paper. It is based on calculating the Cylindrical Modal Coefficients of each individual near-field ring and processing them independently, considering them as cylinders of zero height and applying probe correction. The reconstruction of each far-field cut is obtained through an inverse Fast Fourier Transform. This procedure provides the opportunity to perform real time transformations due to its low acquisition and processing time. It is a useful tool for applications which do not require a full or accurate characterization, such as measuring the main patterns of an antenna or its most important parameters (peak gain, beam width, side lobe level, etc.).
\end{abstract}

Index Terms-Antenna measurement, near-field to far-field transformation, Cylindrical Modal Coefficients, probe correction

\section{INTRODUCTION}

Spherical near-field (SNF) measurement techniques are widely used to characterize the electromagnetic behavior of an antenna. Their development is mainly due to the limitations that are imposed by conventional far-field ranges: electrically large antennas require a vast measuring distance, physically large antennas can suffer from transportation and mounting problems and the influence of the environment can be significant in far-field systems.

However, near-field ranges suffer from the disadvantage that a full sphere needs to be acquired so as to apply a nearfield to far-field transformation, making often the measurement time unaffordable.

In order to perform this transformation, the spherical wave expansion needs to be evaluated in an asymptotic way [1] [2], using the whole sphere data. Since the effect of the probe cannot be neglected when measuring at a close distance from the antenna under test (AUT), probe correction techniques have been developed during the last decades [3]. Besides, some efforts have been made with the aim of enhancing the computational efficiency of the transformation [4].

Antenna manufacturers have a growing interest in the characterization of the main patterns of an AUT. An implementation of a fast and accurate Fresnel zone to far-field algorithm for the main cuts of separable array antennas was introduced in [5], making it possible to determine far-field information of the AUT without measuring a complete sphere.
In this paper, a new fast and computationally efficient approach to reconstruct far-field cuts from measured SNF single rings, applying probe correction, is presented. This method treats each pattern cut independently and is based on the cylindrical modes of a complete ring. Its main advantage is the drastic reduction in the acquisition time, providing the means to obtain the far-field in real time during the course of a measurement.

\section{DESCRIPTION OF THE ALGORITHM}

The transformation algorithm can be divided into three steps, which are depicted in Fig. 1 and are described below.

\section{A. Spherical and cylindrical near-field equivalence}

The starting point is a SNF measurement: either a full sphere or only those cuts that need to be characterized. Each single cut is considered to be independent from the others and is processed apart from them, as an isolated cut of constant $\phi$. A SNF ring can also be seen as a cylindrical near-field (CNF) ring, as it is shown in Fig. 2. The equivalence between them is the following:

$$
\left(E_{z}, E_{\phi}\right)=\left(E_{\phi}, E_{\theta}\right)
$$

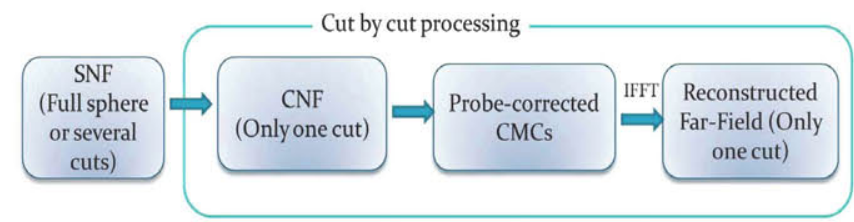

Fig. 1. SNF to FF algorithm outline

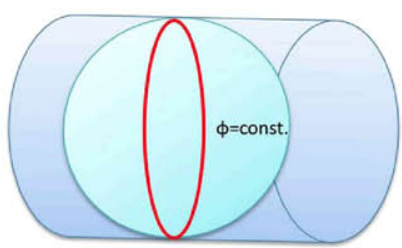

Fig. 2. SNF and CNF ring equivalence 


\section{B. CMCs calculation}

With the aim of having a generic procedure that is valid when measuring with an arbitrary probe, probe-corrected Cylindrical Modal Coefficients (CMCs) are calculated from the CNF cut.

The method to obtain these probe compensated CMCs can be found in [6], considering each cut as a cylinder of zero height, as described in [7]. It can be summarized in three steps:

1) Calculation of the transmitting modal coefficients of the AUT: Instead of performing the 2-Dimensional FFT that would be needed in a general case, a 1-D FFT along $\phi$ is applied, since the height of the cylinder is considered to be zero:

$$
\begin{gathered}
I_{n}(h)=\int_{-\pi}^{\pi} E_{z}\left(r_{0}, \phi\right) \cdot e^{-j n \phi} d \phi \\
I_{n}{ }^{\prime}(h)=\int_{-\pi}^{\pi} E_{\phi}\left(r_{0}, \phi\right) \cdot e^{-j n \phi} d \phi
\end{gathered}
$$

2) Obtention of the probe cylindrical modal coefficients: From the probe far-field pattern, $c_{m}(h)$ and $d_{m}(h)$ are obtained for horizontal polarization and $c^{\prime}{ }_{m}(h)$ and $d_{m}^{\prime}(h)$ for vertical polarization.

3) CMCs calculation: both of the previous coefficients are combined and the Hankel functions are evaluated to calculate the probe-corrected CMCs, with equations (4) and (5):

$$
\begin{aligned}
a_{n}(h)= & \frac{k^{2}}{\Lambda^{2} \Delta_{n}(h)}\left(I_{n}(h) \sum_{m=-\infty}^{\infty} d_{m}{ }^{\prime}(-h) \cdot H_{n+m}{ }^{(2)}\left(\Lambda r_{0}\right)-\right. \\
& \left.-I_{n}{ }^{\prime}(h) \sum_{m=-\infty}^{\infty} d_{m}(-h) \cdot H_{n+m}{ }^{(2)}\left(\Lambda r_{0}\right)\right) \\
b_{n}(h)= & \frac{k^{2}}{\Lambda^{2} \Delta_{n}(h)}\left(I_{n}{ }^{\prime}(h) \sum_{m=-\infty}^{\infty} c_{m}(-h) \cdot H_{n+m}{ }^{(2)}\left(\Lambda r_{0}\right)\right. \\
& \left.-I_{n}(h) \sum_{m=-\infty}^{\infty} c_{m}{ }^{\prime}(-h) \cdot H_{n+m}{ }^{(2)}\left(\Lambda r_{0}\right)\right)
\end{aligned}
$$

where $H_{n+m}{ }^{(2)}\left(\Lambda r_{0}\right)$ is the Hankel function of the second kind, $\mathrm{k}$ is the wave number, $\mathrm{h}$ is any real number and

$$
\begin{gathered}
\Lambda=\left\{\begin{array}{c}
\left(k^{2}-h^{2}\right)^{1 / 2}, h \leq k \\
-j \cdot\left(h^{2}-k^{2}\right)^{1 / 2}, h>k
\end{array}\right. \\
\Delta_{n}(h)=\left(\sum_{m=-\infty}^{\infty} c_{m}(-h) \cdot H_{n+m}{ }^{(2)}\left(\Lambda r_{0}\right)\right) \cdot\left(\sum_{m=-\infty}^{\infty} d_{m}{ }^{\prime}(-h) \cdot H_{n+m}{ }^{(2)}\left(\Lambda r_{0}\right)\right)- \\
-\left(\sum_{m=-\infty}^{\infty} c_{m}{ }^{\prime}(-h) \cdot H_{n+m}{ }^{(2)}\left(\Lambda r_{0}\right)\right) \cdot\left(\sum_{m=-\infty}^{\infty} d_{m}(-h) \cdot H_{n+m}{ }^{(2)}\left(\Lambda r_{0}\right)\right)
\end{gathered}
$$

\section{Far-field reconstruction}

Once the CMCs are calculated, it is easy and fast to reconstruct the Far-field through an inverse Fast Fourier Transform [8].

$$
E(r \rightarrow \infty, \theta, \phi)=-2 k_{0} \sin \theta \sum_{n=-\infty}^{\infty} j^{n} \cdot\left(a_{n} \cdot \hat{\phi}+j \cdot b_{n} \cdot \hat{\theta}\right) \cdot e^{j n \phi}
$$

\section{VALIDATION}

The effectiveness of the method has been verified through measurements from different types of antennas. A comparison is made between our transformation and the SNIFTD (Spherical to far-field transformation by TICRA [9]).

A normalized difference will be calculated to compare the reference SNIFTD pattern $\left({ }^{E_{\text {ref }}}\right)$ with the results obtained with our transformation $\left({ }^{E_{\text {transf }}}\right)$ :

$$
\mathcal{E}=20 \log _{10}\left|10^{E_{\text {ref }} / 20}-10^{E_{\text {trang }} / 20}\right|
$$

where $E_{r e f}$ and $E_{\text {transf }}$ are the far-field amplitudes in $\mathrm{dB}$.

In order to evaluate the accuracy of the algorithm and thus determine if it is acceptable for the application under study, its uncertainty (or error) will be estimated as follows:

$$
\alpha=20 \cdot \log _{10}\left|1-10^{\frac{\varepsilon-m}{20}}\right|
$$

where $\varepsilon$ is the normalized difference obtained with (9) and $\mathrm{m}$ is the far-field pattern cut reconstructed with the proposed method; all of these parameters are expressed in $\mathrm{dB}$.

In this paper, three examples are presented. The first of them is the characterization of the main planes of a horn, an application in which a real time transformation would be very useful. The second one studies a circular polarization array. Lastly, the vertical cut of a BTS antenna is reconstructed.

\section{A. Standard Gain Horn}

In the first experiment, the AUT was chosen to be a MVG standard gain horn SGH820 (Fig. 3) with a working frequency of $10 \mathrm{GHz}$. The acquisition was performed in a MVG StarLab system, with the probes located at a distance of $1 \mathrm{~m}$.

Data from the horizontal and vertical near-field cuts were processed to reconstruct both far-field planes. In Fig. 4 and Fig. 5, a comparison among the acquired near-field and the farfields that are reconstructed with our algorithm and SNIFTD processing can be seen. A good agreement is observed in both planes. 


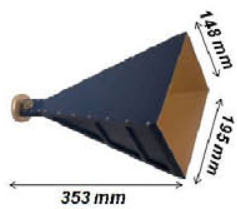

Fig. 3. SATIMO standard gain horn SGH 820

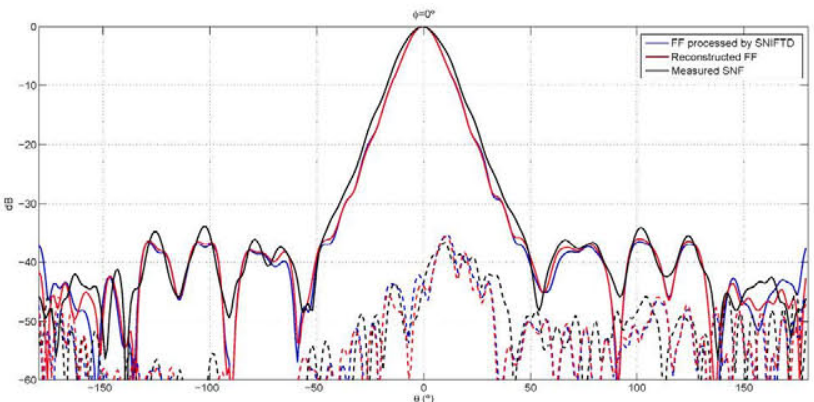

Fig. 4. SGH820 pattern comparison $\left(\phi=0^{\circ}\right)$

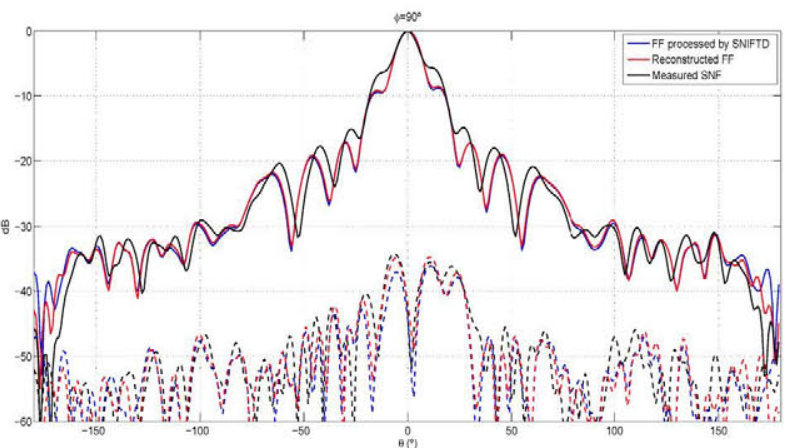

Fig. 5. SGH 820 pattern comparison $\left(\phi=90^{\circ}\right)$

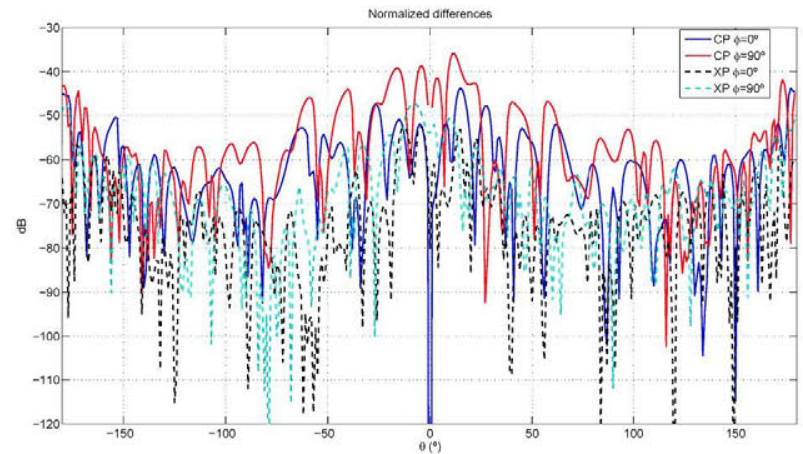

Fig. 6. Normalized differences of the copolar and crosspolar components of the SGH820 main cuts

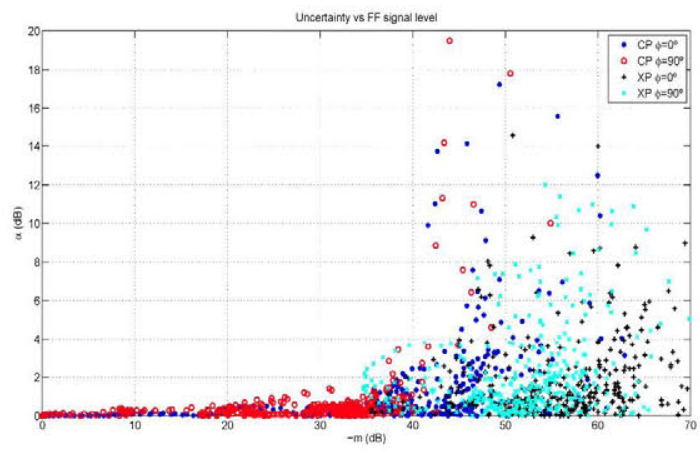

Fig. 7. Uncertainty/Error in the reconstruction of the SGH820 main cuts
In Fig. 6, the normalized differences obtained with (9) are represented for the main cuts. They are always below $-36 \mathrm{~dB}$, showing a good agreement between the reference and the results of our transformation.

An analysis of the variation of the uncertainty or error with the far-field amplitude both for the copolar and crosspolar components is shown in Fig. 7. It is obtained with equation (10). We observe an error below $1 \mathrm{~dB}$ when the signal level is above $-25 \mathrm{~dB}$. The crosspolar component is reconstructed with less accuracy due to its low amplitude.

\section{B. Circular polarization array}

The antenna that was studied in the second test case was a circular polarized array (Fig. 8) with a central frequency of 5.8 $\mathrm{GHz}$. Once again, the acquisition was taken in a StarLab system. The results of the transformation in $\phi=45^{\circ}$ and $\phi=135^{\circ}$ can be observed respectively in Fig. 9 and Fig. 10.

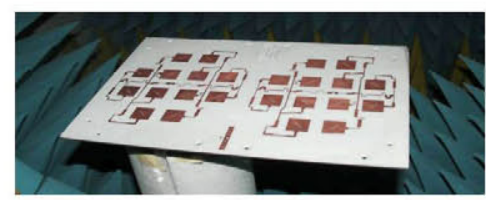

Fig. 8. Circular polarization array

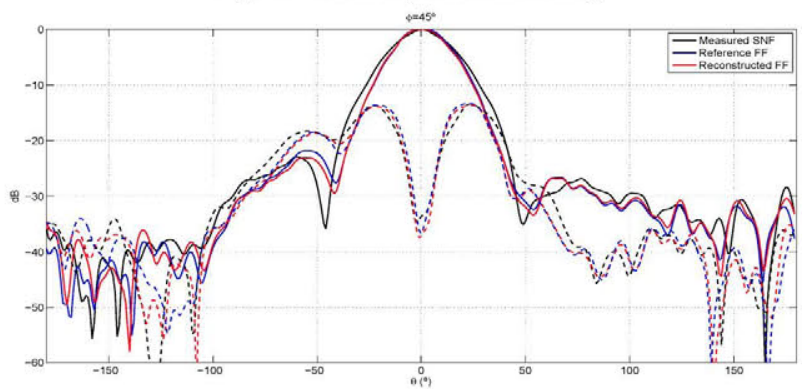

Fig. 9. Array pattern comparison $\left(\phi=45^{\circ}\right)$

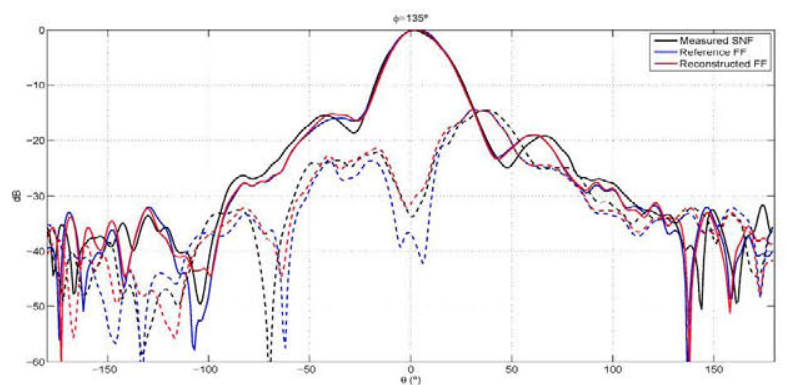

Fig. 10. Array pattern comparison $\left(\phi=135^{\circ}\right)$

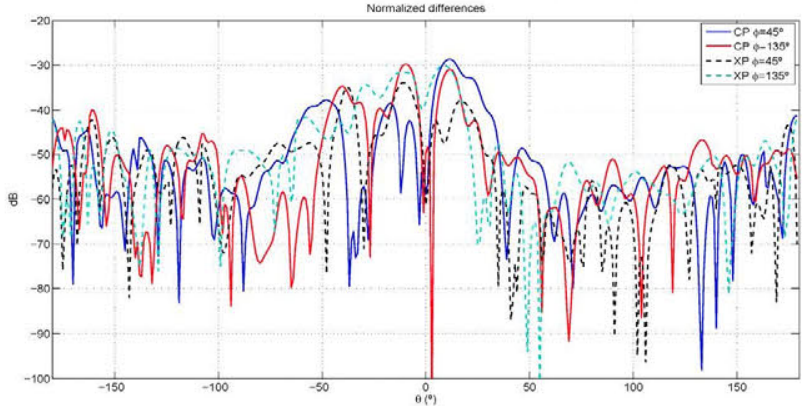

Fig. 11. Normalized differences of the copolar and crosspolar components of the array $\phi=45^{\circ}$ and $\phi=135^{\circ}$ cuts 


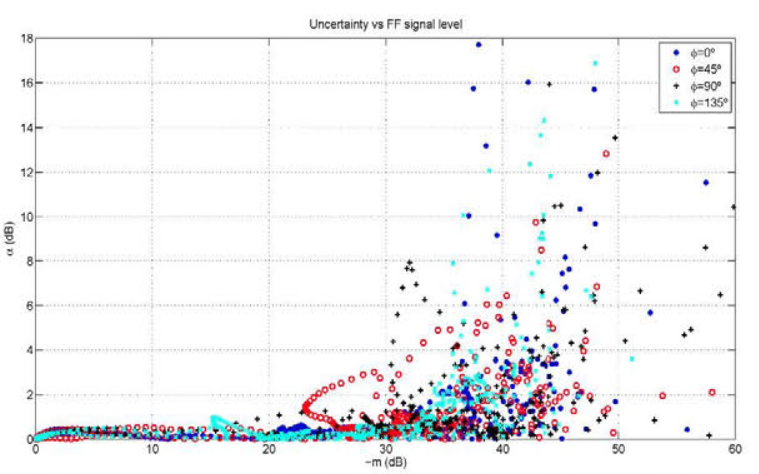

Fig. 12. Uncertainty/Error in the reconstruction of the copolar component in four cuts of the array

Fig. 12 shows the uncertainty (or error) of the transformation in four different cuts. In this case, a similar accuracy is obtained in every plane. Uncertainty is below $1 \mathrm{~dB}$ when the signal level is above $-20 \mathrm{~dB}$ and the normalized differences are below $-30 \mathrm{~dB}$ for the $\phi=45^{\circ}$ and $\phi=135^{\circ}$ cuts (Fig. 11).

\section{BTS Antenna}

Another possible application for this algorithm is the fast characterization of BTS antennas. These antennas usually include several frequency bands, different ports for dual polarization and diverse electrical tilt configurations, making measurement time a critical factor.

The results of applying the single cut transformation algorithm to the vertical plane of a BTS antenna which was measured at the near-field measurement facilities at UPM can be seen in Fig. 13.

As it is shown in Fig. 14, when the vertical plane of the BTS antenna is reconstructed, low normalized differences are obtained. In this case, the difference is lower than $-36 \mathrm{~dB}$ for the copolar field. This is due to the fact that the current sources are enclosed in the line that is defined by the vertical cut. Therefore, this cut contains more information about the field sources and can be reconstructed independently from the others with a higher accuracy.

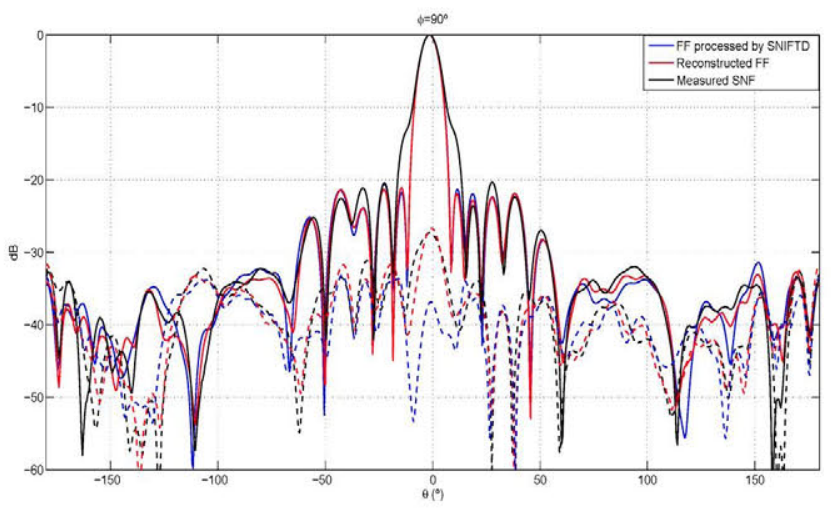

Fig. 13. BTS antenna pattern comparison $\left(\phi=90^{\circ}\right)$

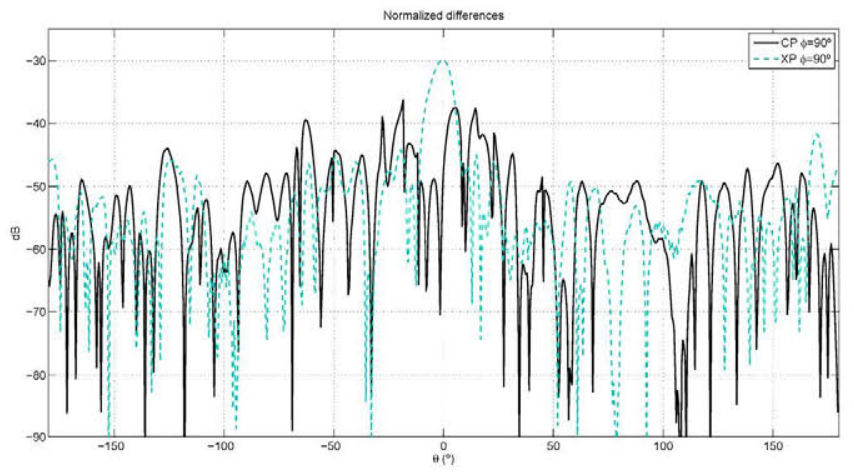

Fig. 14. Normalized differences of the copolar and crosspolar components of the BTS antenna vertical cut

\section{ANALYSIS OF LIMITATIONS}

In general, each far-field cut depends on every spherical near-field cut. Therefore, if a cut by cut transformation is made, some information is lost.

The limitations of this approximation have been analyzed: the dependence of the error on the measuring distance and the cut under study has been verified employing simulation data.

A simulation of a $5 \times 5$ slot array with uniform amplitude and phase excitation at a frequency of $10 \mathrm{GHz}$ is presented in this paper. The 25 slots are equi-spaced in $x$ and $y$, with a separation of $0.67 \lambda$, as it is shown in Fig. 15.

As it can be observed in Fig. 16 and Fig. 17, the best reconstruction is achieved in the cut $\phi=0^{\circ}$ and high errors are obtained in the diagonal cuts.

It has been checked that an accurate reconstruction is achieved when the cut under study encloses a line of field sources and all of them have the same contribution on the acquisition. For example, those cuts in which an array is separable can be reconstructed with a low uncertainty.

Furthermore, it has been verified that the error of the transformation decreases when the measuring distance increases. This behavior is qualitatively shown in Fig. 18.

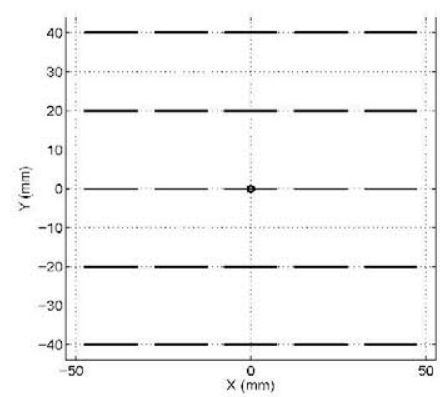

Fig. 15. Simulated slot array 


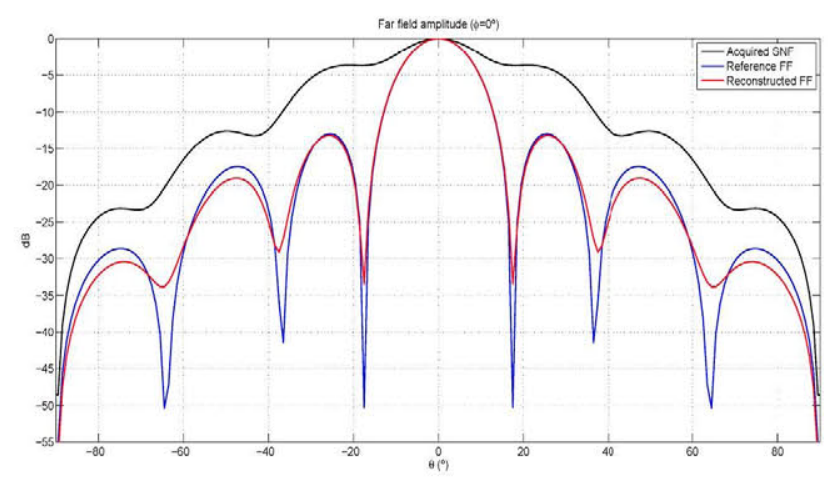

Fig. 16. Simulated slot array copolar pattern comparison $\left(\phi^{=} 0^{\circ}\right)$

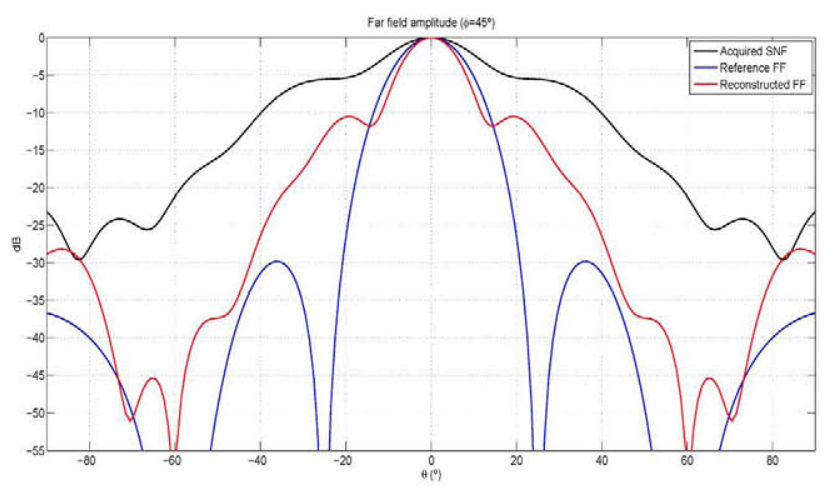

Fig. 17. Simulated slot array copolar pattern comparison $\left(\phi=45^{\circ}\right)$

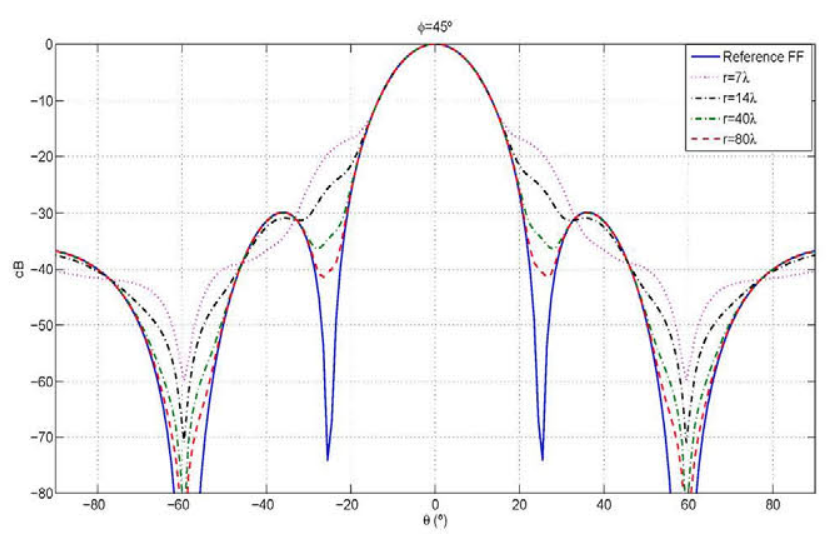

Fig. 18. Variation of the reconstructed copolar far-field with measuring distance

\section{CONCLUSIONS}

The transformation algorithm that has been introduced in this paper is a useful approach to obtain in real time single farfield cuts from spherical near-field rings. It can drastically reduce the measuring time of an AUT that does not require a full characterization

Although information from every spherical near-field cut is generally needed to reconstruct each pattern plane and some information is lost in our approximation, the errors could be neglected for specific situations: in fact, the error of the reconstruction depends on the cut under study and the measuring distance. The best results are achieved when a plane which encloses a line of field sources is analyzed. No information about the antenna geometry is needed and probe correction can be easily applied.

It has been verified through measurements that the far-field reconstruction using cylindrical modes presents a good agreement with the pattern that is processed by SNIFTD. However, it is harder to obtain an accurate crosspolar pattern due to its low level. Several measurements with different antennas under test and different test ranges have been performed to validate the algorithm.

\section{ACKNOWLEDGMENT}

This work has been supported by the SICOMORO project with reference TEC2011-28789-C02-01.

\section{REFERENCES}

[1] A. Yaghjian, "An Overview of Near-Field Antenna Measurements," IEEE Trans. Antennas Propagat., vol. 34, no. 1, pp. 30 45, Jan. 1986

[2] J. E. Hansen, "Spherical Near-Field Antenna Measurements". London, UK: Peter Peregrinus LTd., 1988.

[3] T. Laitinen, S. Pivnenko, and O. Breinbjerg, "Comparison of Probe Correction Techniques for Spherical Near-Field Antenna Measurements," 28th ESA Antenna Workshop on Space Antenna Systems and Technologies, ESTEC, Noordwijk, The Netherlands, May 30 - June 3, pp. 1061-1068, 2005

[4] C. H. Schmidt, T. A Laitinen, Thomas F, Eibert, "Fast Fourier Transform Preprocessing for Accelerated Plane Wave Based Spherical Near-Field Far-Field Transformation", 2010 URSI International Symposium on Electromagnetic Theory, pp. 784-787.

[5] M. Sierra Castañer, S. Burgos, "Fresnel Zone to Far-field Algorithm for Rapid Array Antenna Measurements", Proceedings of the $5^{\text {th }}$ European Conference on Antennas and Propagation (EUCAP), Rome 2011, pp. 3251-3255.

[6] W. M. Leach and D. T. Paris, "Probe compensated near-field measurements on a cylinder", IEEE Transactions on Antennas and Propagation, vol. 21, no. 4, pp. 435-445, Jul. 1973

[7] Fernando Martín Jiménez, “Contribución a las técnicas de medida de arrays planos de gran tamaño para aplicaciones radar", Universidad Politécnica de Madrid, Ph.D. Dissertation, 2009.

[8] O. M. Bucci and C. Gennarelli, "Use of sampling expansions in nearfield-far-field transformations: the cylindrical case," IEEE Trans. Antennas Propagat., vol. 36, no. 6, pp. 830-835, Jun. 1988.

[9] SNIFTD Software by TICRA. www.ticra.com 